\title{
Testing QCD with jets measured by CMS down to very low-pt and into the forward phase space
}

CMS Collaboration, Alex Van Spilbeeck*

University of Antwerp, Belgium

E-mail: alex.vanspilbeeck@uantwerpen.be

\begin{abstract}
The production yields of leading charged-article jets and charged particles in proton-proton collisions are measured at $\sqrt{s}=8 \mathrm{TeV}$ based on a data sample collected with the CMS detector and corresponding to an integrated luminosity of $45 \mu \mathrm{b}^{-1}$. The charged-particle jets (charged particles) are measured in the pseudorapidity range $|\eta|<1.9(2.4)$ for transverse momenta $p_{T}>1(0.8)$ $\mathrm{GeV}$. The measured yields integrated above a given minimum transverse momentum, $p_{T \text { min }}$, provide information on the mechanism by which the underlying parton-parton cross sections are unitarised when approaching the low- $p_{T}$ non-perturbative domain. Comparisons to predictions obtained from various Monte Carlo event generators, show that the measured observable has a large sensitive to the underlying physics.
\end{abstract}

The XXIII International Workshop on Deep Inelastic Scattering and Related Subjects April 27 - May 1, 2015

Southern Methodist University

Dallas, Texas 75275

${ }^{*}$ Speaker. 


\section{Introduction}

The cross section for the production of jets with large transverse momenta in high energy proton-proton ( $\mathrm{pp}$ ) collisions is believed to be derived from scattering of partons, described by perturbative quantum chromodynamics (pQCD). In this framework the partonic cross section is convoluted with the density of partons inside the protons. The measured cross section for the production of jets at large transverse momenta $p_{T}>20 \mathrm{GeV}$ in a pseudorapidity range of $|\eta|<$ 3 , with $\eta=-\ln \left[\tan \left(\frac{\theta}{2}\right)\right]$ and $\theta$ being the polar angle with respect to the anti-clockwise beam direction, is indeed well described by predictions in next-to-leading order QCD [1, 2]. However, most of the final state hadrons produced in pp collisions at the LHC come from the hadronisation of quarks and gluons scattered in (multiple) "semi-hard" interactions with exchanged momenta of $\mathscr{O}(1-3 \mathrm{GeV})$. At such small $p_{T}$ the cross section $\frac{d \sigma}{d p_{T}^{2}} \sim \frac{\alpha_{s}^{2}\left(p_{T}\right)}{p_{T}^{4}}$ is becoming very large and eventually the cross section $\sigma\left(p_{T, \min }\right)=\int_{p_{T, \text { min }}} \frac{d p_{T}^{2} d \sigma}{d p_{T}^{2}}$ exceeds the total inelastic pp cross section. At LHC energies ( $\left.\sigma_{\text {inel }} \approx 70 \mathrm{mb}[3]\right)$ this happens already at $p_{T, \text { min }}$ values of $\mathscr{O}(3 \mathrm{GeV})$, much larger than $\Lambda_{\mathrm{QCD}}[4,5]$. Event generators of hadronic collisions often tame such an infrared divergence with an effective parameter connected to the confinement scale of the hadron [6] such that the parton parton cross section does not exceed the inelastic pp cross section. In addition, at small $p_{T}$ the parton densities are probed in a region where they may be saturared by parton recombination, which will happen even at non-perturbative scales.

The results presented here are based on measurements of track-jets and single tracks and are published in [7]. The two states are complementary since the leading jets are expected to be more correlated to the parton level compared to the leading tracks. On the other hand, the jets are sensitive to the underlying event via a jet pedestal effect, while the leading tracks are not. Trackjets can be defined to very low transverse momenta, and eventually so low that a single track constitutes the jet. The leading tracks are dominated by those hadrons that carry most of the energy of the parent fragmenting parton.

The corrected, integrated leading-jet (charged-particle) $p_{T}$ distribution, normalised by the number of events, is measured as a function of the minimum transverse momentum, $p_{T, \min }$ :

$$
\frac{\sigma\left(p_{T \min }\right)}{\sigma_{\mathrm{vis}}}=\frac{1}{N_{\mathrm{evt}}} \int_{p_{T, \text { min }}} d p_{T, \text { lead }}\left(\frac{d N}{d p_{T, \text { lead }}}\right)
$$

where $N_{\text {evt }}$ is the number of selected events, $\mathrm{N}$ is the number of events with a leading jet (leading charged particle) with a transverse momentum of $p_{T \text {,lead }}$ within $|\eta|<1.9(|\eta|<2.4)$, and $\sigma_{\text {vis }}$ the cross section for all events with a central charged particle with $p_{T, \text { lead }}>0.4 \mathrm{GeV}$.

\section{Experimental setup, event selection and Monte Carlo simulations}

The central feature of the Compact Muon Solenoid (CMS) apparatus is a superconducting solenoid of $6 \mathrm{~m}$ internal diameter, providing a magnetic field of 3.8T. Within the superconducting solenoid volume are a silicon pixel and strip tracker, a lead tungstate crystal electromagnetic calorimeter, and a brass/scintillator hadron calorimeter. Extensive forward calorimetry complements the coverage provided by the barrel and endcap detectors. The inner tracker measures 
charged particles within the pseudorapidity range $|\eta|<2.5$ using silicon pixel and strip detectors located in the magnetic field of the superconducting solenoid. A more detailed description of the CMS detector and the tracker can be found in [8].

The data analysed in this publication, which corresponds to an integrated luminosity of 45 $\mu \mathrm{b}^{-1}$, were collected in July 2012 during a dedicated run with a low probability $(\approx 5.4 \%$ ) of overlapping pp interactions in the same bunch crossing (pile-up). Minimum bias events were triggered by requiring at least one track candidate in either of the TOTEM [10] T2 telescopes, placed symmetrically at $14 \mathrm{~m}$ both sides of the interaction point, covering a pseudorapidity range $5.3<|\eta|<$ 6.5. The analysis presented here follows the procedure described in Ref. [9] where more details on trigger, data selection, pile-up and corrections are given.

For the leading track measurement, events with a leading track in $|\eta|<2.4$ and $p_{T}>400 \mathrm{MeV}$ are selected. These events are used to normalise the integrated distributions in both the track and the jet measurements. Jets are reconstructed offline from tracks with $p_{T}>400 \mathrm{MeV}$ and $|\eta|<2.4$, clustered by the anti- $k_{t}$ algorithm $[11,12,13]$ with a radius parameter of 0.5 . The jet momentum is determined as the vectorial sum of all particle momenta in the jet. A subsample of events with a leading track-jet with $p_{T}>1 \mathrm{GeV}$ and $\left|\eta_{\text {jet }}\right|<1.9$, in addition to the leading track selection defined above, is selected for the leading charged-particle jet analysis.

The data are corrected to stable-particle level, defined to include primary charged particles or decay products with proper lifetimes $(c \tau>1 \mathrm{~cm})$. Events are selected at the stable-particle level if at least 1 charged particle with $p_{T}>40 \mathrm{MeV}$ is present within the range $5.3<|\eta|<6.5$ and at least 1 charged particle with $p_{T}>400 \mathrm{MeV}$ is found within $|\eta|<2.4$. Leading particles are selected as the highest- $p_{T}$ particle from the collection of charged particles within $|\eta|<2.4$ and with $p_{T}>400$ $\mathrm{MeV}$. Jets are clustered from the charged particles with $p_{T}>400 \mathrm{MeV}$ and no restriction in $\eta$, by the anti- $k_{t}$ algorithm with a radius parameter of 0.5 . Leading jets are selected as the highest- $p_{T}$ jet from the collection of charged-particle jets with $p_{T}>1 \mathrm{GeV}$ and $\left|\eta^{\text {jet }}\right|<1.9$.

The Pythia6 [14] (version 6.426) Monte Carlo event generator with tune Z2* and the Pythia 8 [15] (version 8.153) generator with tune 4C are used to correct the data to stable particle level and for physics comparisons. The $\mathrm{Z} 2 *$ tune is derived from the $\mathrm{Z} 1$ tune [16] and updated for the parton density function CTEQ6L [17], with the parameter values for $p_{T}$ cut-off for MPI and its centre-of-mass dependence are changed to $\operatorname{PARP}(82)=1.912$ and $\operatorname{PARP}(90)=0.227$, respectively. The detailed MC simulation of the CMS detector is based on Geant 4 [18]. Simulated events are processed and reconstructed in the same manner as collision data.

The $p_{T}$ distribution of leading track-jets is corrected to stable-particle level applying an iterative unfolding [19] implemented in RooUnfold [20], while for the tracks applying efficiency corrections is sufficient. The response matrix and the efficiency corrections were obtained with Pythia6 and Pythia8. For the final correction the results from the two event generators are averaged. The size of the corrections varies between $5 \%(10 \%)$ at low $p_{T \min }(\sim 1 \mathrm{GeV})$, to $10 \%$ (40\%) at high $p_{T \min }(\sim 45 \mathrm{GeV})$, for the track (jet) measurement.

The normalisation to the total number of selected events is corrected using the ratio between events on the stable-particle level and detector level in the Monte Carlo. This correction factor is 1.11 for Pythia 6 and 1.07 for Pythia 8. The difference is included in the model dependent systematic uncertainty.

Corrections for the $\mathrm{T} 2$ triggered event without a charged primary particle inT2 (fakes) were 

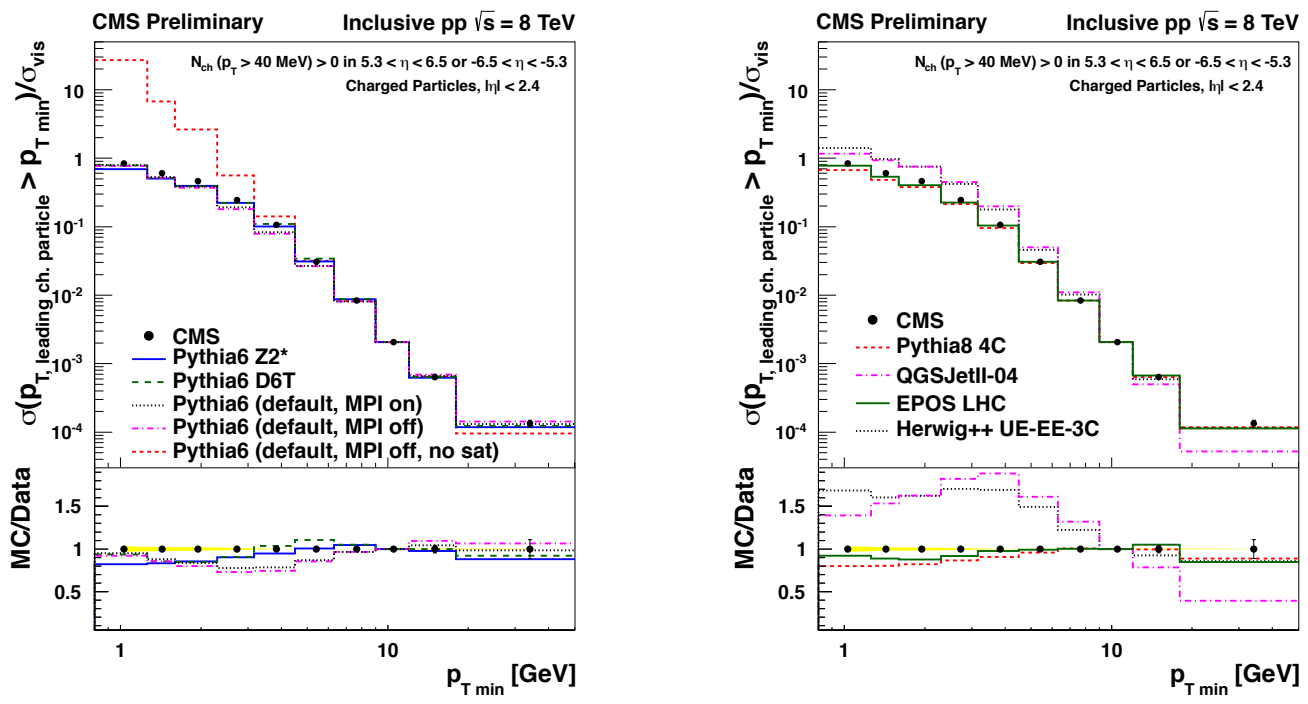

Figure 1: Normalised integrated $p_{T}$ distribution of the leading charged particle in $|\eta|<2.4$. The data are compared to different predictions from various Pythia 6 tunes (left) and various Monte Carlo event generators (right). The error bars indicate the statistical uncertainty and the shaded area the systematic uncertainty. The systematic uncertainties are only shown in the ratio plot. The Monte Carlo curves are normalised to the measured value of $\sigma\left(p_{T \text { lead. char. particle }}>9.0 \mathrm{GeV}\right) / \sigma_{\text {vis }}$. The data points are taken from [9] (included here for completeness and with the same comparison to predictions as for the leading charged particle-jets).

estimated by using Monte Carlo generated events from Pythia 8 and EPOS LHC [21, 22], which have gone through the $\mathrm{T} 2$ dectector simulation. The average corrections for the two models varies from $4 \%(1 \%)$ at low $p_{T \min }(\sim 1 \mathrm{GeV})$ to $7 \%(5 \%)$ at high $p_{T \min }(\sim 45 \mathrm{GeV})$, for the track (jet) analysis. The deviation by Pythia 8 and EPOS is from the average is taken as a model dependent systematic uncertainty (0.1-1.0\%).

The following uncertainties are considered: the uncertainty of $\mathrm{T} 2$ corrections for events without charged primary particle in T2, $0.7 \%(0.1-1.0 \%)$ for the leading track (jet) measurement [9]; the average systematic uncertainty over $p_{T}, \eta$ and $\phi$ associated with track reconstruction efficiency, estimated to be $3.9 \%$; the systematic uncertainty on the unfolding to stable-particle level, which was found to be $2 \%$ in the case of tracks and 2 to $10 \%$ in the case of track-jets, depending on the $p_{T, \text { min }}$ value.

\section{Results}

A subsample of events with a jet with $p_{T}>1 \mathrm{GeV}$ and $\left|\eta_{\text {jet }}\right|<1.9$ is selected for analysis.

The integrated quantity introduced in Eq. (1.1) is obtained experimentally as

$$
\frac{\sigma\left(p_{T \text { lead. }}>p_{T \min }\right)}{\sigma_{\text {vis }}}=\frac{1}{N_{\text {evt }}} \sum_{p_{\text {lead. }}>p_{T \text { min }}} \Delta p_{T \text { lead. }}\left(\frac{\Delta N}{\Delta p_{T \text { lead. }}}\right)
$$



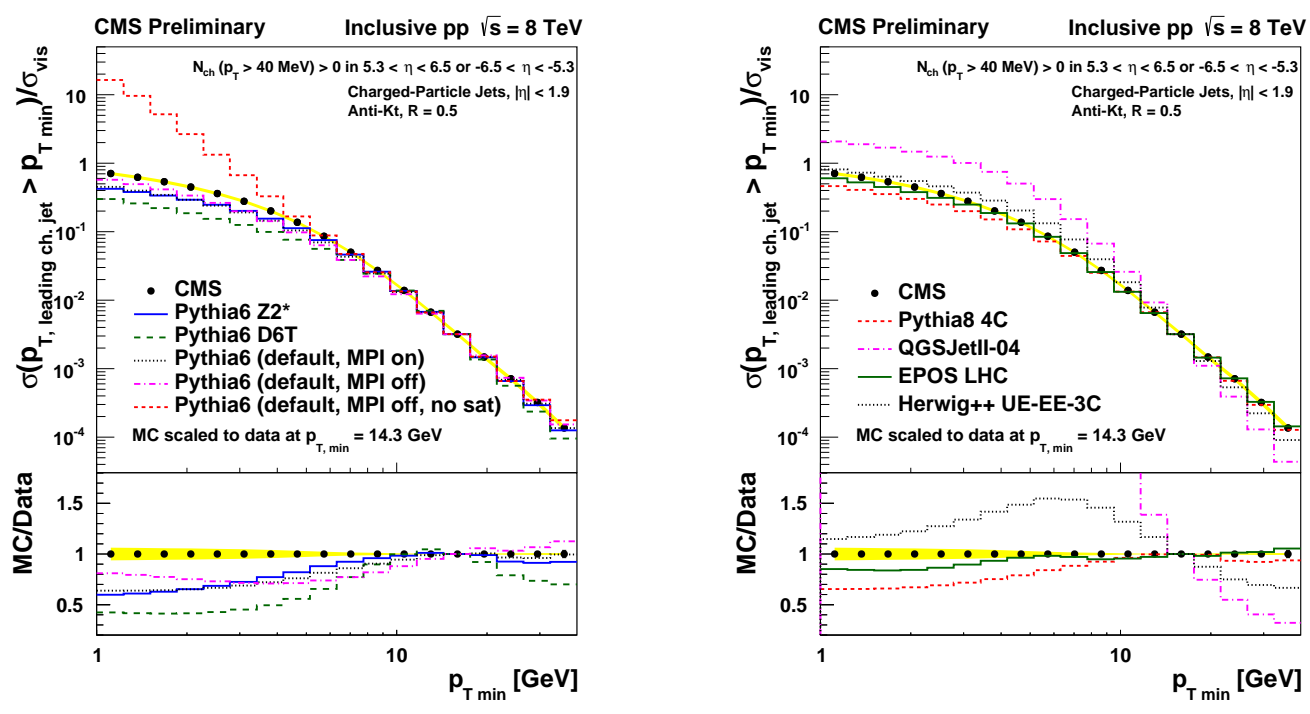

Figure 2: Normalised integrated $p_{T}$ distribution of the leading charged jet in $|\eta|<1$.9. The data are compared to different predictions from various Pythia 6 tunes (left) and various Monte Carlo event generators (right). The error bars indicate the statistical uncertainty and the shaded area the systematic uncertainty. The Monte Carlo curves are normalised to the measured value of $\sigma\left(p_{T \text { lead. char. jet }}>14.3 \mathrm{GeV}\right) / \sigma_{\text {vis. }}$. The uncertainties shown in the ratio plots are thus rescaled with respect to the normalisation of the Monte Carlo predictions.

where $N_{\text {evt }}$ is the number of events with a leading track with $|\eta|<2.4$ and $p_{T}>0.4 \mathrm{GeV}$ and $\Delta p_{T \text { lead }}$ is the bin width of the histogram. In Figs. 1 and 2 the integrated cross section is shown for the leading charged particle (leading charged-particle jet) for $p_{T}>0.8(1 \mathrm{GeV})$ respectively. The data points for leading charged particles (Fig. 1) are taken from Ref. [9] (included here for completeness and with the same comparison to predictions as for the leading charged particle-jets). The distribution falls steeply at at large transverse momenta, and approaches by construction unity at small $p_{T}$. The turn-over from the steeply falling distribution to a flat distribution happens in the range between 1 and $10 \mathrm{GeV}$. However, the exact value where this happens is different for the leading charged particle and the leading charged-particle jet measurement. When jets are clustered, more energy from additional particles is collected within the jet cone. If the jet cone size is reduced, then the leading jet distribution becomes closer to the leading charged-particle distribution.

In the left panels of Figs. 1 and 2 the measured distributions are compared to predictions from the Monte Carlo event generators, Pythia 6 with tune Z2* and D6T, as well as Pythia 6 default with and without MPI. Also shown is the impact of turning off the regularisaton of the cross section completely ("Pythia6 (default, MPI off, no sat)" with $\operatorname{PARP}(81)=\operatorname{PARP}(82)=0$ ). This changes the shape of the distribution by more than an order of magnitude at low $p_{T}$. For illustration, the Monte Carlo predictions are normalised to the measured value of $\sigma\left(p_{T \text { lead. }}>9.0(14.3) \mathrm{GeV}\right) / \sigma_{\text {vis. }}$ for leading charged particles (charged-particle jets).

In the right panels of Figs. 1 and 2 Pythias with tune 4C, Herwig++ (version 2.5.0) 
[23] with tune UE-EE-3C [24] and the Monte Carlo generators used in cosmis ray physics, EPOS [21, 22] LHC tune (based on EPOS 1.99) and QGSJetII-0 4 [25] are shown. The leading track and leading charged jet distributions are best described by EPOS, which deviates only $10 \%$ from the data at lowest $p_{T \min }$ and describes the data at $p_{T \min }>4 \mathrm{GeV}$.

\section{Conclusions}

The normalised cross section for the production of leading charged-particle jets and leading charged particles at small transverse momenta in pp collisions at $\sqrt{s}=8 \mathrm{TeV}$ based on a data sample correponding to an integrated luminosity of $45 \mu \mathrm{b}^{-1}$ collected with the CMS detector is presented. The jets (charged particles) are measured in the pseudorapidity range $|\eta|<1.9$ (2.4) down to transverse momenta $p_{T}=1.0(0.8) \mathrm{GeV}$. The distribution integrated over the transverse momentum as a function of the lower integration boundary, $p_{T, \min }$, falls steeply at large transverse momenta and becomes flatter towards low $p_{T, \min }$, showing the transition from the non-perturbative region.

The behaviour of the cross section from large to small $p_{T}$ has been measured and a significant deviation from the pure perturbative prediction is observed, even at $p_{T}$ values larger than $\Lambda_{\mathrm{QCD}}$. The measured distributions show the transition from the perturbative to the non-perturbative region and indicate that saturation of the parton-parton cross sections is visible already in the $p_{T}$ range of a few $\mathrm{GeV}$ in pp collisions at LHC energies. The transition region is in general not well described by theoretical predictions from various Monte Carlo event generators. The shape of the measured integrated $p_{T}$ distribution is best described by the EPOS generator used in cosmic ray physics.

\section{References}

[1] ATLAS collaboration, "Measurement of inclusive jet and dijet production in $p p$ collisions at $\sqrt{s}=7$ TeV using the ATLAS detector", Phys. Rev. D 86 (2012) 014022, doi:10.1103/PhysRevD.86.014022, arXiv:1112.6297.

[2] CMS collaboration, "Measurements of differential jet cross sections in proton-proton collisions at $\sqrt{s}=7 \mathrm{TeV}$ with the CMS detector", Phys. Rev. D 87 (2013) no. 11, 112002, doi:1103/PhysRevD.87.112002, arXiv:1212.6660.

[3] TOTEM Collaboration, "luminosity-Independent Measurement of the Proton-Proton Total Cross Section at $\sqrt{s}=8 \mathrm{TeV}$ ", Phys. Rev. Lett. 111 (2013), no. 1, 012001, doi:10.1103/PhysRevLettt.111.012001.

[4] T. Sjöstrand and M. van Zijl, "A Multiple Interaction Model for the Event Structure in Hadron Collisions”, Phys. Rev. D 36 (1987) 2019, doi : 10.1103 /Phys RevD . 36.2019.

[5] T. Sjöstrand and P.Z. Skands, "Multiple interactions and the structure of beam remnants", JHEP $\mathbf{0 3}$ (2004) 053, doi:10.1088/1126-6708/2004/03/053.

[6] J. Dischler and T. Sjöstrand, "A Toy model of color screening in the proton”, Eur. Phys. J. C. 3 (2001) , arXiv: hep-ph/0011282.

[7] CMS Collaboration, "Production of leading charged particles and leading charged-particle jets at small transverse momenta in pp collisions at $\sqrt{s}=8 \mathrm{TeV}$, $\operatorname{arXiv}: 1507.00233$. 
Leading Charged Particle (Jet) Cross Sections at Small Transverse Momenta in pp Collisions at $\sqrt{s}=8 \mathrm{TeV}$ Alex Van Spilbeeck

[8] CMS Collaboration, “Track and vertex reconstruction in CMS”, Nucl. Instr. Meth. A 582 (2007) 781-784, doi:10.1088/1748-0221/3/08/S08804.

[9] CMS collaboration, "Pseudorapidity and leading transverse momentum distributions of charged particles in pp collisions at $8 \mathrm{TeV}$ ", CMS-PAS-FSQ-12-026 (2013).

[10] TOTEM Collaboration, "The TOTEM Experiment at the CERN Large Hadron Collider", JINST 3 (2008) S08007, doi:10.1088/1748-0221/3/08/S08007.

[11] M. Cacciari and G. P. Salam, 'Dispelling the $N^{3}$ myth for the $k_{t}$ jet-finder'”, Phys. Lett. B 641 (2006) 57-61, doi:10.1016/j.physletb.2006.08.037, arXiv:hep-ph/0512210.

[12] M. Cacciari, G. P. Salam, and G. Soyez, 'The anti- $k_{t}$ jet clustering algorithm'”, JHEP 04 (2008) 063 , doi:10.1088/1126-6708/2008/04/063, arXiv:0802.1189.

[13] M. Cacciari, G. P. Salam, and G. Soyez, "FastJet User Manual”, Eur. Phys. J. C 72 (2012) 1986, doi:10.1140/epjc/s10052-012-1896-2, arXiv:1111.6097.

[14] T. Sjöstrand, S. Mrenna, and P. Z. Skands, "PYTHIA 6.4 physics and manual", JHEP 05 (2006) 026, doi:10.1088/1126-6708/2006/05/026, arXiv:hep-ph/0603175.

[15] T. Sjöstrand, S. Mrenna, and P. Z. Skands, "A Brief Introduction to PYTHIA 8.1", Comput.Phys.Commun. 178 (2008) 852-867, doi:10.1016/j.cpc.2008.01.036, arXiv:0710.3820.

[16] R. Field, "Early LHC Underlying Event Data - Findings and Surprises", (2010), arXiv: 1010.3558.

[17] J. Pumplin, D. R. Stump, J. Huston, H.L. Lai, P. Nadolsky and W. K. Tunk, "New generation of parton distributions with uncertainties from global QCD analysis”, JHEP 07 (2002) 012 , doi:10.1088/1126-6708/2002/07/012, arXiv:hep-ph/0201195.

[18] GEANT4 Collaboration, "GEANT4: A Simulation toolkit”, Nucl. Instrum. Meth. A 506 (2003) 250-303, doi:10.1016/S0168-9002 (03) 01368-8.

[19] G. D’Agostin, “A Multidimensional unfolding method based on Bayes' theorem”, Nucl. Instrum. Meth. A362 (1995) 487-498, doi : $10.1016 / 0168-9002$ (95) $00274-\mathrm{X}$.

[20] T. Adye, "Unfolding algorithms and tests using RooUnfold", Proceedings of the PHYSTAT 2011 Workshop, CERN, Geneva, Switzerland, January 2011, CERN-2011-006, pp.313-318, arXiv:1105.1160.

[21] K. Werner, F.-M. Liu, and T. Pierog, "Parton ladder splitting and the rapidity dependence of transverse momentum spectra in deuteron-gold collisions at RHIC", Phys.Rev. C 74 (2006) 044902, doi:10.1103/PhysRevC.74.044902, arXiv:hep-ph/0506232.

[22] T. Pierog et al., "EPOS LHC : test of collective hadronization with LHC data", arXiv: 1306.0121.

[23] M. Bahr, S. Gieseke, M. A. Gigg, D. Grellscheid, K. Hamilton, O. Latunde-Dada, S. Platzer, P. Richardson, M. H. Seymour, A. Sherstnev, J. Tully and B. R. Webber, "Herwig++ Physics and Manual”, Eur. Phys. J. C58 (2008) 639-707, doi:10.1140/epjc/s10052-008-0798-9, arXiv:0803.0883.

[24] S. Gieseke, D. Grellscheid, K. Hamilton, A. Papaefstathiou, S. Plätzer, P. Richardson, C. A. Rhr, P. Ruzicka, A. Sidmok, L. Suter and D. Winn, "Herwig++ 2.5 Release Note", arXiv: 1102.1672.

[25] S. Ostapchenko, "Monte Carlo treatment of hadronic interactions in enhanced Pomeron scheme: I. QGSJET-II model”, Phys.Rev. D 83 (2011) 014018, doi : 10.1103 / PhysRevD . 83. 014018 , arXiv:1010.1869. 